\title{
REVIEW ARTICLE Regional tissue oxygenation monitoring in the neonatal intensive care unit: evidence for clinical strategies and future directions
}

\author{
Jonathan P. Mintzer ${ }^{1}$ and James E. Moore ${ }^{2}$
}

\begin{abstract}
Near-infrared spectroscopy (NIRS)-based monitoring of regional tissue oxygenation $\left(\mathrm{rSO}_{2}\right)$ is becoming more commonplace in the neonatal intensive care unit (NICU). While increasing evidence supports $\mathrm{rSO}_{2}$ monitoring, actual standards for applying this noninvasive bedside technique continue to evolve. This review highlights the current strengths and pitfalls surrounding practical NIRS-based monitoring in the neonatal population. The physiologic background of $\mathrm{rSO}_{2}$ monitoring is discussed, with attention to understanding oxygen delivery/consumption mismatch and its effects on tissue oxygen extraction. The bedside utility of both cerebral and peripheral $\mathrm{rSO}_{2}$ monitoring in the NICU is then explored from two perspectives: (1) disease/event-specific "responsive" monitoring and (2) "routine," continuous monitoring. Recent evidence incorporating both monitoring approaches is summarized with emphasis on practical applicability in the NICU. Finally, a future paradigm for a broad-based NIRS monitoring strategy is presented, with attention towards improving personalization of neonatal care and ultimately enhancing long-term outcomes.
\end{abstract}

Pediatric Research (2019) 86:296-304; https://doi.org/10.1038/s41390-019-0466-9

\section{INTRODUCTION}

Assessment of regional tissue oxygenation $\left(\mathrm{rSO}_{2}\right)$ utilizing nearinfrared spectroscopy (NIRS) is becoming more common in the neonatal intensive care unit (NICU). ${ }^{1-10}$ While normative studies are required to delineate expected NIRS values and signal behavior in neonates, evidence supporting the potential utility of NIRS monitoring is growing. Currently, cerebral $\mathrm{rSO}_{2}$ monitoring is most prevalent in the neonatal literature; however, recent studies continue to advance the use of peripheral NIRS monitoring. ${ }^{4,9,11-16}$ This review aims to highlight current evidence and assist in structuring the bedside use of noninvasive NIRSbased $\mathrm{rSO}_{2}$ monitoring in the NICU setting.

\section{BACKGROUND}

NIRS provides regional hemoglobin oxygenation status using a technique similar to pulse oximetry $\left(\mathrm{SpO}_{2}\right)$. Both forms of monitoring take advantage of differential absorption spectra between oxygenated and deoxygenated hemoglobin to visible light in the near-infrared range. ${ }^{1,4,9,17-21}$ This difference is then expressed as a ratio [(oxyhemoglobin/oxyhemoglobin+deoxyhemoglobin) $\times 100$ ] for real-time trending. The primary contrast between $\mathrm{SpO}_{2}$ and NIRS involves hemoglobin oxygenation data processing prior to display on bedside monitors. ${ }^{1,4,9,17}$

In pulse oximetry, hemoglobin oxygenation data are reported solely from pulsatile, or arterialized, sources with non-pulsatile sources (e.g., venous, capillary) mathematically subtracted from the data output stream. ${ }^{18,22}$ Peripheral pulsatile vessels conveying $\mathrm{SpO}_{2}$ data are anatomically defined as arteries originating from the aorta. Therefore, as arteries typically contain highly oxygenated blood, $\mathrm{SpO}_{2}$ represents a global estimate of precapillary oxygen delivery to peripheral tissues. ${ }^{17,22}$

In contrast to pulse oximetry, NIRS expresses full tissue hemoglobin oxygenation without subtraction of non-pulsatile data. Thus, NIRS represents the regional oxygenated to total hemoglobin ratio $\left(\mathrm{rSO}_{2}\right)$ for the combined arterial, capillary, and venous hemoglobin sources underlying a given sensor. ${ }^{1,4,5,9,10,14,17,23-26}$ Anatomically, at any given time, the blood contained within an individual tissue segment exists in a generally accepted vascular distribution of approximately $20 \%$ arterial, $75 \%$ venous, and 5\% capillary. ${ }^{9,25,27,28}$ Therefore, as NIRS provides predominantly post-capillary tissue oxygenation information, it may be considered a surrogate estimate of local tissue oxygen utilization.

The utility of NIRS in clinical practice may be considered in relation to the theoretical critical oxygen delivery point concept. ${ }^{1,29,30}$ According to this postulate, tissue oxygen utilization remains relatively constant across a range of oxygen delivery with oxygen extraction stable to meet tissue metabolic requirements. Initially, decreases in oxygen delivery produce subtle increases in oxygen extraction to maintain tissue homeostasis. However, with further oxygen deprivation, a threshold is crossed ("the critical $\mathrm{O}_{2}$ point") beyond which ongoing tissue oxygen utilization becomes oxygen delivery-dependent. Below the critical $\mathrm{O}_{2}$ point, dramatic increases in oxygen extraction are theorized as necessary to maintain tissue metabolic needs. This circumstance is often accompanied by clinical signs consistent with oxygen delivery-consumption mismatch (see Fig. 1). ${ }^{1,30}$

Integration of $\mathrm{SpO}_{2}$ and NIRS data allows for crude estimation of tissue oxygen extraction. Fractional tissue oxygen extraction

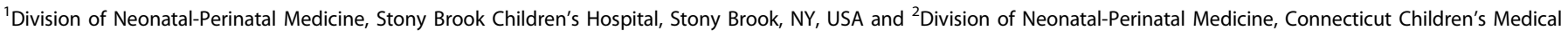
Center, Hartford, CT, USA

Correspondence: Jonathan P. Mintzer (jmintzermd@gmail.com)

Received: 14 December 2018 Revised: 24 May 2019 Accepted: 8 June 2019

Published online: 27 June 2019 


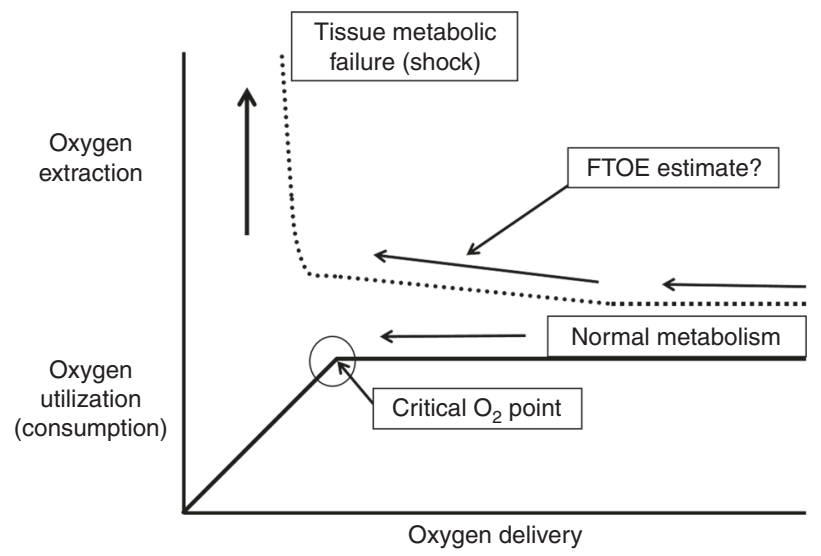

Fig. 1 Theoretical relationship described between oxygen delivery and both oxygen utilization/consumption and oxygen extraction

(FTOE) is calculated as follows: $:^{8,9,31-41}$

$\mathrm{FTOE}=\left[\left(\mathrm{SpO}_{2}-\right.\right.$ regionalrSO 2$\left.) / \mathrm{SpO}_{2}\right]$

In this approach, the numerator represents local tissue oxygen extraction, while the $\mathrm{SpO}_{2}$ in the denominator converts this extraction into a function based on oxygen delivery. While common in clinical studies, FTOE cannot distinguish $\mathrm{rSO}_{2}$ changes resulting from diminished tissue perfusion from those occurring due to increased tissue oxygen extraction/utilization. However, it is feasible that FTOE trending may provide noninvasive clinical decision support at the bedside.

\section{CURRENT STATE OF EVIDENCE}

Dozens of examples of NIRS monitoring in the NICU setting have been described in the literature. ${ }^{3,8-10,15,17,42,43}$ While cerebral monitoring is most frequently studied, peripheral monitoring of renal, splanchnic, and/or peripheral muscle $\mathrm{rSO}_{2}$ has been reported as well. In these reports, a wide range of NIRS monitoring practices have been described as potential adjuncts in the management of an equally broad number of neonatal conditions. ${ }^{5,8-11,16,44-47}$ These data have highlighted the potential utility of "oxygenation adequacy" monitoring with a goal of improving neonatal outcomes. ${ }^{1,3,8}$ However, in a recent survey of NIRS uptake and usage among NICU clinicians, concerns remain regarding actual evidence-based clinical utility among the general NICU population. ${ }^{48}$

Currently, cerebral $\mathrm{rSO}_{2}$ monitoring has evolved into routine practice in certain limited scenarios. Over the past decade, the neonatal neurocritical care paradigm, incorporating cerebral imaging, amplitude-integrated electroencephalography, and cerebral NIRS, has evolved for management of neonates at high risk for neurologic disorders. ${ }^{4,34,49-55}$ Additionally, the SafeBoosC studies have examined the practicality and utility of cerebral $\mathrm{rSO}_{2}$ monitoring and guideline-driven targeting on clinical outcomes among extremely premature infants. ${ }^{56-62}$ Ongoing research into these paradigms remains an area of highly active inquiry.

In contrast, no specific framework currently exists to routinely incorporate both cerebral and peripheral NIRS monitoring in neonatal critical care. ${ }^{3,5,9,54}$ Interestingly, given the physiologic redundancy of cerebral perfusion, along with well-described neuroprotective blood flow redistribution during neonatal distress, one can argue that peripheral $\mathrm{rSO}_{2}$ monitoring may be more sensitive than cerebral for demonstrating acute perturbations in physiologic oxygenation homeostasis. ${ }^{27,37}$ Synthesizing these NIRS monitoring practices into a coherent bedside strategy remains a work in progress.

In general, there are two approaches to NIRS monitoring in the NICU setting. In this review, disease- or event-specific monitoring, referred to as "responsive" monitoring, is defined as goal-oriented use of NIRS for specific clinical or bedside purposes. The second approach, referred to as "routine" monitoring, concerns the continuous use of NIRS in a framework similar to current bedside cardiopulmonary vital sign monitoring practices. The remainder of this review is dedicated to delineating the potential benefits and pitfalls of these neonatal NIRS monitoring strategies.

\section{"RESPONSIVE" MONITORING}

The "responsive" $\mathrm{rSO}_{2}$ monitoring paradigm concerns the use of NIRS to generate specific information for particular clinical management decisions. In this decision-support framework, responsive monitoring may also be considered as a method to gauge clinical responses to therapeutic maneuvers. ${ }^{1,5,8-10}$ This form of NIRS monitoring has also been periodically used to examine the effects of routine NICU care practices on tissue oxygen utilization. In this section, we will review evidence regarding responsive NIRS monitoring on the clinical evaluation of anemia, patent ductus arteriosus, and feeding intolerance. We will additionally highlight examples of NIRS-based procedural monitoring to demonstrate the responsive paradigm.

\section{Anemia}

NIRS monitoring has been utilized in numerous studies to evaluate the central and peripheral oxygen extraction and utilization effects of anemia. These studies generally report baseline central and peripheral $\mathrm{rSO}_{2}$ and/or FTOE followed by responses to transfusion. As expected, packed red blood cell transfusion, by increasing global oxygen carrying capacity, results in decreased oxygen extraction evidenced by a rise in regional $\mathrm{rSO}_{2}{ }^{32,36,63-73}$ Interestingly, these responses to transfusions, which knowingly increase both overall blood volume and the concentration of chromophores per unit blood volume, are tissue specific, with peripheral tissues often demonstrating a more robust response compared to the brain. ${ }^{36,37,63,66}$

Using transfusion responses as a guide, current research seeks to derive anemia-specific cerebral and/or somatic $\mathrm{rSO}_{2}$ or FTOE patterns to assist in transfusion practices. ${ }^{1,10,37,73,74}$ In 2002, Wardle et al. $^{70}$ conducted a study using elevated forearm FTOE as a transfusion threshold, though data were skewed by transfusions provided for clinical concerns within the NIRS-based transfusion group. Defining FTOE-based transfusion thresholds remains a work in progress, especially as oxygen extraction behavior in anemia differs in a tissue-specific manner. ${ }^{36,37,63,66,73}$ It appears that NIRS monitoring can provide important information for transfusion management, although specific practices remain elusive at present.

In addition, several reports have identified NIRS-based patterns as potentially predisposing factors for transfusion-related acute gut injury, or necrotizing enterocolitis occurring in close contiguity following blood transfusion. In some reports, reductions in splanchnic NIRS and/or increases in signal variability have been associated with necrotizing enterocolitis following packed red blood cell transfusion. ${ }^{75-78}$ However, another study demonstrated no differences in NIRS parameters following transfusion. ${ }^{72}$ Larger studies into the relationship between blood transfusions and splanchnic oxygenation are required to resolve these disparate findings.

Hemodynamically significant patent ductus arteriosus The evaluation of suspected hemodynamically significant patent ductus arteriosus (hsPDA) poses an interesting avenue of inquiry for neonatal NIRS monitoring. Physiologically, ductal flow across 
an hsPDA is inferred to cause pulmonary overcirculation at the expense of systemic flow. ${ }^{79-81}$ Currently, management of hsPDA remains a highly controversial issue in neonatology. ${ }^{79,80,82-88}$ Indeed, the definition of hemodynamic significance remains contested at present. $^{79,81}$ In addition, agreed-upon specific echocardiographic parameters to define hsPDA remain undefined. ${ }^{89}$ Finally, adjunctive PDA-related management, including fluid intake practices, also controversial, limit the generalizability of PDA-related studies. ${ }^{81,90}$ Given the above, the utility of NIRS within hsPDA management has yet to be clearly defined.

Several studies have assessed whether NIRS monitoring may aid in determining the hemodynamic significance of PDA-related shunting. While some reports have demonstrated physiologically plausible $\mathrm{rSO}_{2}$ decreases and/or FTOE increases in the presence of an hsPDA, these findings have not been consistent across studies. ${ }^{91-95}$ In addition, responses to both medical and surgical hsPDA therapies have been evaluated for their effects on tissue oxygenation and oxygen extraction. ${ }^{96-99}$

Whether cerebral and/or peripheral NIRS monitoring may aid in PDA management remains speculative. Moreover, significant differences exist between cerebral and somatic oxygenation in the context of hsPDA, predominantly accounted for by cerebral autoregulation. ${ }^{91}$ Further evaluation of peripheral NIRS monitoring is required to determine potential sensitivity compared to cerebral $\mathrm{rSO}_{2}$ as a marker for tissue oxygenation failure in the context of hsPDA. Given the physiology of hsPDA-related shunting and its plausible effects on tissue oxygen delivery/consumption balance, future larger-scale studies with standardized PDA-related definitions and NIRS data collection practices are needed.

Feeding intolerance/necrotizing enterocolitis

Numerous reports have been published on the potential utility of splanchnic NIRS monitoring for the evaluation of feeding intolerance and necrotizing enterocolitis. ${ }^{11,16,100}$ Common to these studies is a splanchnic observational data set, often paired with cerebral monitoring, that demonstrates changes occurring during various feeding regimens. Moreover, several reports have evaluated changes in splanchnic NIRS data as a potential warning sign for future development of necrotizing enterocolitis (NEC), although with widely variable results. ${ }^{78,100-104}$ Importantly, these numerous papers highlight the difficulty of analyzing real-time splanchnic monitoring data, predominantly due to inherent signal variability.

For example, initial reports demonstrated daily mean splanchnic $\mathrm{rSO}_{2}$ and FTOE values in premature neonates with expected changes occurring during the first two postnatal weeks. ${ }^{105}$ This same study reported decreased splanchnic $\mathrm{rSO}_{2}$ and increased FTOE in infants with feeding intolerance and further noted persistently low $\mathrm{rSO}_{2}$ with low variability as a potential risk factor for NEC, a finding replicated in a larger prospective observational study. ${ }^{103}$ In another report, splanchnic NIRS monitoring was able to aid in distinguishing surgically complicated NEC from uncomplicated cases, but not differentiating the absence of NEC from active disease. ${ }^{104}$

Other studies have reported correlations between splanchnic NIRS and superior mesenteric artery Doppler values with promising results. ${ }^{101,106,107}$ While splanchnic NIRS may provide valuable information on intestinal perfusion, these correlative data cannot take into account changes in tissue oxygen demand, for example as a result of feeding. Furthermore, recent work has demonstrated a positive correlation between intestinal peristaltic activity and splanchnic $\mathrm{rSO}_{2}$ in premature neonates, with implications for prospective assessment of feeding tolerance. ${ }^{108}$

Additional studies have investigated responses to various feeding regimens, including continuous vs. bolus feeding, ${ }^{109}$ and breast milk vs. formula feeding. ${ }^{110}$ Splanchnic $\mathrm{rSO}_{2}$ data has also been reported to correlate with early feeding intolerance in premature neonates, both using continuous and bolus feeding regimens. ${ }^{111-113}$ Similar reports have focused on the intrauterine growth restriction population given the high incidence of feeding intolerance in this group. ${ }^{114}$

When viewed as a series, these studies suggest a pressing need for further inquiry. Importantly, splanchnic NIRS monitoring data is often highly variable at baseline, with frequent signal drop-out. Inherent splanchnic $\mathrm{rSO}_{2}$ quiescent variability has been reported as approaching $25 \%$, with differences in variability depending on how raw splanchnic data are averaged. ${ }^{27}$ Abdominal sensor placement, specifically overlying the liver vs. the infraumbilical position, can affect splanchnic NIRS monitoring values and variability. ${ }^{115}$ It thus follows that feeding- or NEC-related changes in splanchnic $\mathrm{rSO}_{2}$ need to be carefully assessed in the context of this variability. Future work is crucial to define appropriate data management techniques to account for this variability in addition to assessing comparisons between splanchnic and other, less variable monitoring sites.

\section{Procedure-related monitoring}

Tissue $\mathrm{rSO}_{2}$ monitoring can demonstrate subtle changes in tissue oxygen utilization behavior, often before the onset of clinical symptoms. ${ }^{1,8-10}$ Therefore, one can argue that NIRS monitoring could provide bedside information on factors related to routine NICU care. Currently, studies on specific procedure-related monitoring are most common in the perioperative environment. ${ }^{116-118}$ However, NICU procedure-related monitoring has demonstrated some interesting results that warrant further investigation.

For example, several reports have demonstrated decreases in cerebral oxygenation following umbilical arterial blood drawing. ${ }^{119-123}$ In one study across a broad range of gestational ages, cerebral $\mathrm{rSO}_{2}$ decrements were attributed to faster blood draws. $^{123}$ In another report specific to very low birth weight neonates, larger volumes of blood draws were more likely to be associated with cerebral $\mathrm{rSO}_{2}$ decreases. ${ }^{122}$ In a further study on very low birth weight neonates, the common occurrence and natural course of these decrements was demonstrated, with an additional observation of a prolonged cerebral $\mathrm{rSO}_{2}$ recovery time in some neonates. ${ }^{120}$ Finally, a follow-up study on cerebral $\mathrm{rSO}_{2}$ monitoring demonstrated cerebral decrements occurring most commonly among neonates with lower baseline $\mathrm{SpO}_{2}, \mathrm{PaO}_{2}$, and cerebral $\mathrm{rSO}_{2}$, thus insinuating subject-related factors as contributing to sensitivity to this common procedure. ${ }^{119}$

Additional studies have assessed the relationship between neonatal head and/or body positioning and tissue $\mathrm{rSO}_{2}$ parameters. In one study evaluating a series of different body positions in premature neonates, no position-related cerebral $\mathrm{rSO}_{2}$ differences were observed. ${ }^{124}$ Similar findings were also observed for both cerebral and mesenteric $\mathrm{rSO}_{2}$ in clinically stable very low birth weight neonates following both pre- and postprandial body position changes. ${ }^{125}$ Two additional studies have employed cerebral NIRS monitoring to demonstrate that responses to head-tilting maneuvers, as indicators of neonatal cerebrovascular control, differ between premature and term neonates, ${ }^{126}$ and in response to prone positioning. ${ }^{127}$

These reports indicate the versatile nature of NIRS monitoring among the neonatal population. As familiarity with this monitoring technique increases, further publications will likely demonstrate unique and interesting uses of $\mathrm{rSO}_{2}$ monitoring. While NIRS monitoring could conceivably allow for more personalized, patient-centered approaches to NICU care, whether this modality will ultimately improve short- and long-term outcomes remains unknown at this time.

\section{"ROUTINE" MONITORING}

The "routine" NIRS monitoring approach involves $\mathrm{rSO}_{2}$ monitoring as a continuous, trendable metric used in routine neonatal 
Table 1. Comparisons of NIRS-based oxygenation studies

Birth transition studies

\begin{tabular}{|c|c|c|c|c|c|c|}
\hline Year & Author & $n$ & Population & Age & Sites & Data epochs \\
\hline 2010 & Bernal et al. ${ }^{166}$ & 26 & Term (not specified) & $2-8 \mathrm{~h}$ & Cerebral renal & $1 \mathrm{~min}$ \\
\hline 2013 & Pichler et al. ${ }^{38}$ & 381 & $\begin{array}{l}\text { Term-vaginal }(40 \pm 1.3 \text { weeks GA) } \\
\text { Term-CS }(39 \pm 0.9 \text { weeks GA) } \\
\text { Preterm-CS }(34.9 \pm 1.4 \text { weeks GA) }\end{array}$ & $0-15 \mathrm{~min}$ & Cerebral & $1 \mathrm{~min}$ \\
\hline 2015 & Baik et al. $^{167}$ & 140 & Term $(38.8 \pm 0.9$ weeks GA) & $0-15 \min$ & Cerebral & $1 \mathrm{~min}$ \\
\hline 2015 & Montaldo et al. ${ }^{129}$ & 61 & $\geq 37$ weeks GA & $0-9 \mathrm{~h}$ & Cerebral renal splanchnic & $1 \mathrm{~min}$ \\
\hline
\end{tabular}

Longer term studies

\begin{tabular}{llllllc}
\hline Year & Author & $n$ & Population & Age & Monitoring sites & Data epochs \\
\hline 2010 & McNeill et al. $^{132}$ & 12 & $29-34$ weeks GA & $0-21$ days & Cerebral renal splanchnic & $24 \mathrm{~h}$ \\
2010 & Cortez et al. $^{105}$ & 19 & $\leq 30$ weeks GA & $0-14$ days & Splanchnic & $24 \mathrm{~h}$ \\
2014 & Bailey et al. $^{131}$ & 38 & $37-42$ weeks GA & $8-48 \mathrm{~h}$ & Cerebral renal splanchnic & $1 \mathrm{~h}$ \\
2014 & ${\text { Patel et al. }{ }^{103}}$ & 92 & $<32$ weeks GA and $<1500 \mathrm{~g} \mathrm{BW}$ & $0-4$ weeks & Splanchnic & 5 min single recordings \\
2016 & Alderliesten et al. $^{31}$ & 999 & $<32$ weeks GA & $0-72 \mathrm{~h}$ & Cerebral & $1 \mathrm{~h}$ \\
2018 & Elsayed et al. $^{14}$ & 32 & $30 \pm 3$ weeks GA & $31 \pm 14$ days & Cerebral renal splanchnic & 10 min \\
\hline
\end{tabular}

NIRS near-infrared spectroscopy, CS C-section, GA gestational age, $B W$ body weight

practice. In this paradigm, cerebral or peripheral $\mathrm{rSO}_{2}$ and/or FTOE is considered as an additional bedside vital sign. As above, several studies have demonstrated that NIRS-specific changes often occur prior to the onset of clinically noticeable sequelae. At present, however, whether this monitoring strategy can function as a realtime early-warning tool has yet to be determined. In this section, we will review important information and pitfalls concerning the signal variability observed in central and peripheral $\mathrm{rSO}_{2}$ monitoring. We will also explore some current approaches utilizing routine neonatal NIRS monitoring, including neurocritical monitoring and perioperative management. Finally, we will review correlations between $\mathrm{rSO}_{2}$ data and invasive monitoring data.

\section{Normative data/variability}

Several studies have reported reference ranges for both cerebral and peripheral $\mathrm{rSO}_{2}$ among neonates. These studies include both fullterm and premature neonates and, taken together, provide data on cerebral, renal, and splanchnic $\mathrm{rSO}_{2}$. Furthermore, while most studies focus on short-term NIRS monitoring (e.g., during birth transition), $38,53,128-130$ others have sought to demonstrate normative NIRS ranges over longer postnatal intervals. ${ }^{14,31,103,105,131,132}$

In addition to establishing normative ranges, the inherent variability within individual $\mathrm{rSO}_{2}$ data streams has been described, although in small studies. ${ }^{14,27,52,53}$ In most studies in which cerebral and peripheral NIRS monitoring is performed, variability differences between NIRS signals have been observed. In Table 1, selected studies are displayed to demonstrate the varied approaches that have been used in managing $\mathrm{rSO}_{2}$ information as a continuous data stream. Determining how to account for normal baseline variability ("signal noise") within NIRS data sets remains as yet undefined, especially with regard to splanchnic monitoring. ${ }^{11,16,27}$ Clearly, future studies are required to standardize data collection and management techniques to properly evaluate the use of NIRS as a routine monitoring technique.

Additional attention to splanchnic NIRS monitoring is specifically warranted in this regard. As above, multiple studies have examined the potential utility of splanchnic NIRS monitoring, especially regarding feeding intolerance and necrotizing enterocolitis. ${ }^{11,16}$ However, whether splanchnic variability results from actual changes in mesenteric oxygen delivery/consumption balance vs. abdominal sensor placement over a hollow anatomic cavity has yet to be conclusively determined. Until larger-scale, standardized normative NIRS data are obtained to address this quandary, careful critical attention is required in assessing the actual bedside utility of splanchnic $\mathrm{rSO}_{2}$ monitoring.

Neurocritical monitoring/cerebral autoregulation

Over recent years, cerebral $\mathrm{rSO}_{2}$ monitoring has been incorporated into the neurocritical monitoring paradigm. In this approach, ongoing neurologic assessment is performed for neonates at highest risk for neurodevelopmental impairment. In addition to neurologic imaging and amplitude-integrated electroencephalography, changes in cerebral oxygenation are considered in managing this vulnerable population. ${ }^{52,53,133}$

Continuous, routine cerebral $\mathrm{rSO}_{2}$ monitoring has also been used to describe cerebral autoregulation in neonates, or an ability to maintain stable cerebral perfusion across a range of blood pressure. ${ }^{2,43,134,135}$ Furthermore, several reports have described decreases in cerebral autoregulatory capacity, or pressure-passive circulation, as an indicator of worsening clinical status among neonates. ${ }^{50,118,134,136,137}$ Moreover, these NIRS-based changes may be observable earlier than evidence of clinical deterioration, thus promoting careful cerebral $\mathrm{rSO}_{2}$ monitoring as a potential earlywarning tool. ${ }^{51,138-140}$ Although certainly promising, further inquiry is needed to assess how to best incorporate this strategy into routine practice and whether actual neurodevelopmental outcomes can be improved with this approach. ${ }^{4,23,42,54,135,141,142}$

Attempting to maintain cerebral oxygenation within a set range has also been an active area of inquiry. ${ }^{143}$ In the original SafeBoosC observational trial, investigators demonstrated the feasibility of maintaining preterm neonates within a predefined cerebral range $(55-85 \%){ }^{144}$ Subsequently, the SafeBoosC-II trial randomized extremely premature neonates to either guidelinebased cerebral NIRS targeting using the above range vs. NIRS monitoring with no targeting. ${ }^{56,57}$ In the SafeBoosC-II trial, subjects in the targeting group were successfully maintained within range compared to the control group, ${ }^{44}$ with NIRS-based alarms guiding interventions in approximately $25 \%$ of cases. ${ }^{62}$ Thus, an increased number of bedside interventions occurred in the NIRS targeting 
group, with uncertain impact. However, this feasibility study was not powered for clinical outcomes. Further analyses demonstrated no statistically significant differences between groups with regard to cranial ultrasound and magnetic resonance imaging (MRI) evidence of brain injury; ${ }^{60}$ amplitude-integrated electroencephalography and brain injury-related biomarkers; 58,61 or 2-year neurodevelopmental outcomes. ${ }^{59}$ A further study, adequately powered for clinical outcomes, is currently being planned to comprehensively assess the cerebral $\mathrm{rSO}_{2}$ targeting approach among premature neonates (SafeBoosC-III; see http://www. safeboosc.eu).

Cerebral $\mathrm{rSO}_{2}$ targeting can also be considered from a "pattern recognition" framework. ${ }^{10}$ In this approach, $\mathrm{rSO}_{2}$ targeting is based on the appearance of departures from baseline on longitudinal monitoring. At present, cerebral $\mathrm{rSO}_{2}$ and $\mathrm{FTOE}$ baseline values have been best described among the preterm population during the first 72 postnatal hours. ${ }^{31}$ In this paradigm, careful multimodal analysis is performed to determine and act upon the most likely etiology of cerebral oxygen delivery/ consumption imbalance (e.g., hypo/hyperoxia, hypo/hypercapnia, anemia, hypotension, hypoglycemia, intracranial hemorrhage, and impaired cerebral autoregulation). Although further study is needed, this pattern recognition-based paradigm appears promising as a likely future practice to incorporate routine NIRS monitoring into NICU care.

\section{Perioperative and ECMO monitoring}

One of the most commonly reported uses of NIRS monitoring in the neonatal population occurs in the perioperative and extracorporeal membrane oxygenation (ECMO) setting. Particularly, cerebral and occasionally peripheral $\mathrm{rSO}_{2}$ monitoring are frequently utilized in the context of congenital heart disease repair both intraoperatively and in postoperative management. In the operating room, NIRS-based evaluation of tissue oxygen delivery/ consumption balance has become a fairly standard adjunctive vital sign used to aid in optimizing tissue perfusion during surgery and/or ECMO. ${ }^{117,145-147}$

In addition, perioperative $\mathrm{rSO}_{2}$ monitoring has demonstrated efficacy in early recognition of low perfusion states with important implications for operative morbidity and mortality. ${ }^{136,139,148-151}$ Furthermore, postoperative cerebral $\mathrm{rSO}_{2}$ monitoring has been observed to correlate with short-term lactates and both survival and neurodevelopmental outcomes following cardiac surgery. ${ }^{116,152-155}$ In addition, changes in both intraoperative and postoperative renal $\mathrm{rSO}_{2}$ parameters have been shown to correlate with later development of acute kidney injury. ${ }^{156,157}$ Splanchnic monitoring has additionally been investigated as potentially associated with the development of necrotizing enterocolitis among postoperative cardiac surgery patients. ${ }^{102}$ Finally, cerebral $\mathrm{rSO}_{2}$ monitoring has been utilized in neonates undergoing ECMO support, ${ }^{158}$ with one study demonstrating a correlation between cerebral $\mathrm{rSO}_{2}$ values and overall survival in this population. ${ }^{145}$

Perioperative NIRS monitoring has also been described following non-cardiac procedures. ${ }^{159}$ For example, cerebral and renal $\mathrm{rSO}_{2}$ have been reported as providing useful information during and following alimentary tract procedures, ${ }^{160}$ although specific management recommendations remain lacking. In addition, intraoperative cerebral monitoring was described as providing clinically actionable information allowing for respiratory adjustments during esophageal atresia repair. ${ }^{161,162}$

While standardization and best practices have yet to be formally derived, these examples offer insight into the possible benefits of both central and peripheral NIRS monitoring among critically ill neonates. Further research is required to aid in extrapolating the experience of perioperative NIRS monitoring to NICU-specific practices.

\section{Correlational analyses}

Several reports have demonstrated correlations between conventional monitoring data and tissue-specific NIRS-based parameters. These small-scale observational studies have investigated these relationships both in the NICU and among the operative and ECMO populations. Although larger, standardized studies are required to further investigate these relationships, the available data do support the physiologic plausibility underlying NIRS monitoring.

For example, in an observational analysis of cerebral NIRS, both $\mathrm{rSO}_{2}$ and FTOE correlated with heart rate, respiratory rate, and pulse oximetry among 30 to 42 weeks gestational age neonates during the first six postnatal hours. ${ }^{163}$ However, no correlation was observed between cerebral NIRS and arterial oxygenation in a separate study. ${ }^{164}$ Interestingly, in another study, cerebral FTOE, although not pulse oximetry, was found to be associated with severe retinopathy of prematurity among preterm neonates $<30$ weeks gestation. ${ }^{41}$ In addition, significant correlations were observed between both cerebral and splanchnic $\mathrm{rSO}_{2}$ and FTOE with mean blood pressure in a small cohort of neonates ranging from 22 to 42 weeks gestational age. ${ }^{35}$ In another study, renal $\mathrm{rSO}_{2}$ was shown to directly correlate with urine output among a cohort of neonates on ECMO for congenital diaphragmatic hernia. ${ }^{146}$ Finally, cerebral $\mathrm{rSO}_{2}$ has been observed to correlate with brain MRI-based regional cerebral blood flow values among neonates with hypoxic-ischemic encephalopathy. ${ }^{165}$

Other studies have sought to determine correlations between invasive monitoring parameters and NIRS-based values. In one study, cerebral and renal FTOE were observed to inversely correlate with hematocrit level, although a similar relationship could not be demonstrated for splanchnic oxygenation likely due to excessive signal variability. ${ }^{37}$ In addition, as previously discussed, physiologically plausible responses to packed red blood cell transfusion further imply a correlation between $\mathrm{rSO}_{2}$ and FTOE values and degree of anemia. However, at present, organ-specific FTOE-based transfusion thresholds have not yet been defined. ${ }^{73,74}$ Although beyond the scope of this NICUspecific review, it is worth mentioning that several studies have reported plausible correlations between tissue oxygenation values and invasive monitoring parameters among the pediatric and adult operative and intensive care populations.

\section{FUTURE DIRECTION/VISION}

Tissue oxygenation monitoring offers an interesting and objective perspective on a neonate's overall clinical status. Using this noninvasive approach, physiologic changes in tissue oxygen delivery/utilization behavior can be observed in real time. However, as we have described in detail, carefully designed broad-based future studies are required to maximize the potential of this monitoring approach.

The routine monitoring approach as described above requires familiarity with normal tissue-specific oxygenation behavior. Comparing NIRS monitoring to pulse oximetry, there is a broad difference in experience regarding the perception of "normal" between these two monitoring approaches. Moreover, when to intervene and what to expect in response to a pulse oximetrybased intervention is already well understood. Despite numerous studies, this is not necessarily the case for NIRS monitoring at this time.

One concern is that currently available NIRS monitoring devices report raw $\mathrm{rSO}_{2}$ data in short intervals with trends typically available as a horizontal graph. As numerous factors affect realtime $\mathrm{rSO}_{2}$, with individual tissues demonstrating unique oxygenation behavior, the practicality of using real-time raw $\mathrm{rSO}_{2}$ signals in bedside NICU care is challenging. An interesting improvement would be the addition of pulse oximetry data to NIRS devices. This 
would allow more rapid analysis of a given perturbation in $\mathrm{rSO}_{2}$ signals along with allowing real-time tissue-specific FTOE trending.

In addition, NIRS signals are much more variable than pulse oximetry. It is therefore difficult to determine in real-time whether an individual $\mathrm{rSO}_{2}$ departure from baseline represents an actual clinical event vs. a momentary change. Thus, it is currently uncertain whether real-time raw $\mathrm{rSO}_{2}$ displays on NIRS devices are the best method to report this tissue-specific behavior. Another advance could have future devices displaying a more integrated NIRS profile on-screen. Such a display would include raw NIRS (and/or FTOE) data along with short- and longer-term trends to allow for enhanced contextualization and improved clinicaldecision support.

Most importantly, large-scale studies are required to establish a normative data set, including region-specific baseline variability, among the overall NICU population. To accomplish this goal, a dedicated research consortium may be considered to assemble these data in a reference registry from which normative analyses may be performed. Discovering the true potential of routine $\mathrm{rSO}_{2}$ monitoring depends on the establishment of agreed-upon norms, standards, and expectations.

\section{CONCLUSION}

Current NICU monitoring practices are primarily centered around indirect measures, including cardiorespiratory vital signs and serum markers of impaired oxygen delivery/consumption balance. NIRS monitoring, on the other hand, offers measured direct realtime tissue oxygen extraction/utilization behavior in a noninvasive monitor. While studies have shown potential uses for NIRS monitoring in the NICU, elucidating specific strategies, best practices, and effects on long-term outcomes remains a work in progress.

\section{AUTHOR CONTRIBUTIONS}

J.P.M. contributed to initial article conceptualization, outline/framework, initial drafting, and editing/finalization for publication. J.E.M. contributed to initial article conceptualization, topic selection, overall manuscript design, and editing/finalization for publication. Both authors agree with publication of the manuscript in its current form.

\section{ADDITIONAL INFORMATION}

Competing interests: The authors declare no competing interests.

Publisher's note: Springer Nature remains neutral with regard to jurisdictional claims in published maps and institutional affiliations.

\section{REFERENCES}

1. Andersen, C. C., Hodyl, N. A., Kirpalani, H. M. \& Stark, M. J. A theoretical and practical approach to defining "adequate oxygenation" in the preterm newborn. Pediatrics 139, e20161117 (2017).

2. Caicedo, A. et al. A new framework for the assessment of cerebral hemodynamics regulation in neonates using NIRS. Adv. Exp. Med. Biol. 876, 501-509 (2016).

3. El-Khuffash, A. \& McNamara, P. J. Hemodynamic assessment and monitoring of premature infants. Clin. Perinatol. 44, 377-393 (2017).

4. Escourrou, G. et al. How to assess hemodynamic status in very preterm newborns in the first week of life? J. Perinatol. 37, 987-993 (2017).

5. Evans, K. M. \& Rubarth, L. B. Investigating the role of near-infrared spectroscopy in neonatal medicine. Neonatal Netw. 36, 189-195 (2017).

6. Garvey, A. A., Kooi, E. M. W., Smith, A. \& Dempsey, E. M. Interpretation of cerebral oxygenation changes in the preterm infant. Children (Basel) 5, 94 (2018).

7. Kenosi, M., Naulaers, G., Ryan, C. A. \& Dempsey, E. M. Current research suggests that the future looks brighter for cerebral oxygenation monitoring in preterm infants. Acta Paediatr. 104, 225-231 (2015).

8. Korcek, P., Stranak, Z., Sirc, J. \& Naulaers, G. The role of near-infrared spectroscopy monitoring in preterm infants. J. Perinatol. 37, 1070-1077 (2017).
9. Sood, B. G., McLaughlin, K. \& Cortez, J. Near-infrared spectroscopy: applications in neonates. Semin. Fetal Neonatal Med. 20, 164-172 (2015).

10. van Bel, F. \& Mintzer, J. P. Monitoring cerebral oxygenation of the immature brain: a neuroprotective strategy? Pediatr. Res. 84, 159-164 (2018).

11. Bailey, S. M. \& Mally, P. V. Review of splanchnic oximetry in clinical medicine. J. Biomed. Opt. 21, 091306 (2016).

12. Cerbo, R. M. et al. Global perfusion assessment and tissue oxygen saturation in preterm infants: where are we? Early Hum. Dev. 89(Suppl. 1), S44-S46 (2013).

13. Chock, V. Y., Frymoyer, A., Yeh, C. G. \& Van Meurs, K. P. Renal saturation and acute kidney injury in neonates with hypoxic-ischemic encephalopathy undergoing therapeutic hypothermia. J. Pediatr. 200, 232-239e1 (2018).

14. Elsayed, Y. N. et al. Integrated evaluation of hemodynamics: a novel approach for the assessment and management of preterm infants with compromised systemic circulation. J. Perinatol. 38, 1337-1343 (2018).

15. Marin, T. \& Moore, J. Understanding near-infrared spectroscopy. Adv. Neonatal Care 11, 382-388 (2011).

16. Martini, S. \& Corvaglia, L. Splanchnic NIRS monitoring in neonatal care: rationale, current applications and future perspectives. J. Perinatol. 38, 431-443 (2018).

17. Chakravarti, S., Srivastava, S. \& Mittnacht, A. J. C. Near infrared spectroscopy (NIRS) in children. Semin. Cardiothorac. Vasc. Anesth. 12, 70-79 (2008).

18. Chan, E. D., Chan, M. M. \& Chan, M. M. Pulse oximetry: understanding its basic principles facilitates appreciation of its limitations. Respir. Med. 107, 789-799 (2013).

19. Fouzas, S., Priftis, K. N. \& Anthracopoulos, M. B. Pulse oximetry in pediatric practice. Pediatrics 128, 740-752 (2011).

20. McMorrow, R. C. \& Mythen, M. G. Pulse oximetry. Curr. Opin. Crit. Care 12 269-271 (2006).

21. Sinex, J. E. Pulse oximetry: principles and limitations. Am. J. Emerg. Med. 17, 59-67 (1999).

22. Jubran, A. Pulse oximetry. Crit. Care 19, 272 (2015).

23. Hyttel-Sorensen, S., Greisen, G., Als-Nielsen, B. \& Gluud, C. Cerebral near-infrared spectroscopy monitoring for prevention of brain injury in very preterm infants. Cochrane Database Syst. Rev. 9, CD011506 (2017).

24. Toet, M. C. \& Lemmers, P. M. Brain monitoring in neonates. Early Hum. Dev. 85 77-84 (2009).

25. Watzman, H. M. et al. Arterial and venous contributions to near-infrared cerebral oximetry. Anesthesiology 93, 947-953 (2000).

26. Wolfberg, A. J. \& du Plessis, A. J. Near-infrared spectroscopy in the fetus and neonate. Clin. Perinatol. 33, 707-728 (2006).

27. Mintzer, J. P., Parvez, B., Chelala, M., Alpan, G. \& LaGamma, E. F. Quiescent variability of cerebral, renal, and splanchnic regional tissue oxygenation in very low birth weight neonates. J. Neonatal Perinatal Med. 7, 199-206 (2014).

28. Scott, J. P. \& Hoffman, G. M. Near-infrared spectroscopy: exposing the dark (venous) side of the circulation. Paediatr. Anaesth. 24, 74-88 (2014).

29. Andersen, C. C. et al. The cerebral critical oxygen threshold of ventilated preterm lambs and the influence of antenatal inflammation. J. Appl. Physiol. 2011, 775-781 (1985)

30. Ronco, J. J. et al. Identification of the critical oxygen delivery for anaerobic metabolism in critically ill septic and nonseptic humans. JAMA 270, 1724-1730 (1993).

31. Alderliesten, T. et al. Reference values of regional cerebral oxygen saturation during the first 3 days of life in preterm neonates. Pediatr. Res. 79, 55-64 (2016).

32. Andersen, C. C., Karayil, S. M., Hodyl, N. A. \& Stark, M. J. Early red cell transfusion favourably alters cerebral oxygen extraction in very preterm newborns. Arch. Dis. Child Fetal Neonatal Ed. 100, F433-F435 (2015).

33. Baenziger, O., Keel, M., Bucher, H. U. \& Wolf, M. Oxygen extraction index measured by near infrared spectroscopy-a parameter for monitoring tissue oxygenation? Adv. Exp. Med. Biol. 645, 161-166 (2009).

34. Balegar, K. K., Stark, M. J., Briggs, N. \& Andersen, C. C. Early cerebral oxygen extraction and the risk of death or sonographic brain injury in very preterm infants. J. Pediatr. 164, 475-480 e1 (2014).

35. Massa-Buck, B., Amendola, V., McCloskey, R. \& Rais-Bahrami, K. Significant correlation between regional tissue oxygen saturation and vital signs of critically ill infants. Front. Pediatr. 5, 276 (2017).

36. Mintzer, J. P., Parvez, B., Chelala, M., Alpan, G. \& LaGamma, E. F. Monitoring regional tissue oxygen extraction in neonates $<1250 \mathrm{~g}$ helps identify transfusion thresholds independent of hematocrit. J. Neonatal Perinatal Med. 7, 89-100 (2014).

37. Mintzer, J. P., Parvez, B. \& La Gamma, E. F. Regional tissue oxygen extraction and severity of anemia in very low birth weight neonates: a pilot NIRS analysis. Am. J. Perinatol. 35, 1411-1418 (2018).

38. Pichler, G. et al. Reference ranges for regional cerebral tissue oxygen saturation and fractional oxygen extraction in neonates during immediate transition after birth. J. Pediatr. 163, 1558-1563 (2013).

39. Rallis, D. et al. Evaluation of cerebral oxygenation in neonates with sepsis with near-infrared spectroscopy. Am. J. Perinatol. 34, 419-427 (2017). 
40. Vesoulis, Z. A., Liao, S. M. \& Mathur, A. M. Gestational age-dependent relationship between cerebral oxygen extraction and blood pressure. Pediatr. Res. 82, 934-939 (2017).

41. Vesoulis, Z. A., Lust, C. E., Liao, S. M., Trivedi, S. B. \& Mathur, A. M. Early hyperoxia burden detected by cerebral near-infrared spectroscopy is superior to pulse oximetry for prediction of severe retinopathy of prematurity. J. Perinatol. 36, 966-971 (2016).

42. da Costa, C. S., Greisen, G. \& Austin, T. Is near-infrared spectroscopy clinically useful in the preterm infant? Arch. Dis. Child Fetal Neonatal Ed. 100, F558-F561 (2015).

43. Kooi, E. M. W. et al. Measuring cerebrovascular autoregulation in preterm infants using near-infrared spectroscopy: an overview of the literature. Expert Rev. Neurother. 17, 801-818 (2017).

44. Hyttel-Sorensen, S. et al. Cerebral near infrared spectroscopy oximetry in extremely preterm infants: phase II randomised clinical trial. BMJ 350, g7635 (2015).

45. Pichler, G. et al. Avoiding arterial hypotension in preterm neonates (AHIP)-a single center randomised controlled study investigating simultaneous near infrared spectroscopy measurements of cerebral and peripheral regional tissue oxygenation and dedicated interventions. Front. Pediatr. 6, 15 (2018).

46. Smallwood, C. D. \& Walsh, B. K. Noninvasive monitoring of oxygen and ventilation. Respir. Care 62, 751-764 (2017).

47. van Bel, F., Lemmers, P. \& Naulaers, G. Monitoring neonatal regional cerebral oxygen saturation in clinical practice: value and pitfalls. Neonatology 94, 237-244 (2008)

48. Hunter, C. L., Oei, J. L., Suzuki, K., Lui, K. \& Schindler, T. Patterns of use of nearinfrared spectroscopy in neonatal intensive care units: international usage survey. Acta Paediatr. 107, 1198-1204 (2018).

49. Ancora, G., Maranella, E., Locatelli, C., Pierantoni, L. \& Faldella, G. Changes in cerebral hemodynamics and amplitude integrated EEG in an asphyxiated newborn during and after cool cap treatment. Brain Dev. 31, 442-444 (2009).

50. Chalak, L. F., Tarumi, T. \& Zhang, R. The "neurovascular unit approach" to evaluate mechanisms of dysfunctional autoregulation in asphyxiated newborns in the era of hypothermia therapy. Early Hum. Dev. 90, 687-694 (2014).

51. Chalak, L. F. \& Zhang, R. New wavelet neurovascular bundle for bedside evaluation of cerebral autoregulation and neurovascular coupling in newborns with hypoxic-ischemic encephalopathy. Dev. Neurosci. 39, 89-96 (2017).

52. El-Dib, M. et al. Brain maturity and variation of oxygen extraction in premature infants. Am. J. Perinatol. 33, 814-820 (2016).

53. El-Dib, M. et al. EEG maturation and stability of cerebral oxygen extraction in very low birth weight infants. J. Perinatol. 36, 311-316 (2016).

54. Gumulak, R., Lucanova, L. C. \& Zibolen, M. Use of near-infrared spectroscopy (NIRS) in cerebral tissue oxygenation monitoring in neonates. Biomed. Pap. Med Fac. Univ. Palacky. Olomouc Czech Repub. 161, 128-133 (2017).

55. ter Horst, H. J., Verhagen, E. A., Keating, P. \& Bos, A. F. The relationship between electrocerebral activity and cerebral fractional tissue oxygen extraction in preterm infants. Pediatr. Res. 70, 384-388 (2011).

56. Hyttel-Sorensen, S. et al. A phase II randomized clinical trial on cerebral nearinfrared spectroscopy plus a treatment guideline versus treatment as usual for extremely preterm infants during the first three days of life (SafeBoosC): study protocol for a randomized controlled trial. Trials 14, 120 (2013).

57. Pellicer, A. et al. The SafeBoosC phase II randomised clinical trial: a treatment guideline for targeted near-infrared-derived cerebral tissue oxygenation versus standard treatment in extremely preterm infants. Neonatology 104, 171-178 (2013).

58. Plomgaard, A. M. et al. Early biomarkers of brain injury and cerebral hypo- and hyperoxia in the SafeBoosC II trial. PLoS ONE 12, e0173440 (2017).

59. Plomgaard, A. M. et al. No neurodevelopmental benefit of cerebral oximetry in the first randomised trial (SafeBoosC II) in preterm infants during the first days of life. Acta Paediatr. https://doi.org/10.1111/apa.14463 (2018).

60. Plomgaard, A. M. et al. Brain injury in the international multicenter randomized SafeBoosC phase II feasibility trial: cranial ultrasound and magnetic resonance imaging assessments. Pediatr. Res. 79, 466-472 (2016).

61. Plomgaard, A. M. et al. The SafeBoosC II randomized trial: treatment guided by near-infrared spectroscopy reduces cerebral hypoxia without changing early biomarkers of brain injury. Pediatr Res. 79, 528-535 (2016)

62. Riera, J. et al. The SafeBoosC phase II clinical trial: an analysis of the interventions related with the oximeter readings. Arch. Dis. Child Fetal Neonatal Ed. 101, F333-F338 (2016).

63. Bailey, S., Hendricks-Muñoz, K., Wells, J. \& Mally, P. Packed red blood cell transfusion increases regional cerebral and splanchnic tissue oxygen saturation in anemic symptomatic preterm infants. Am. J. Perinatol. 27, 445-453 (2010).

64. Banerjee, J., Leung, T. S. \& Aladangady, N. Effect of blood transfusion on intestinal blood flow and oxygenation in extremely preterm infants during first week of life. Transfusion 56, 808-815 (2016).
65. Banerjee, J., Leung, T. S. \& Aladangady, N. Cerebral blood flow and oximetry response to blood transfusion in relation to chronological age in preterm infants. Early Hum. Dev. 97, 1-8 (2016).

66. Dani, C., Pratesi, S., Fontanelli, G., Barp, J. \& Bertini, G. Blood transfusions increase cerebral, splanchnic, and renal oxygenation in anemic preterm infants. Transfusion 50, 1220-1226 (2010).

67. Sandal, G. et al. Assessment of red blood cell transfusion and transfusion duration on cerebral and mesenteric oxygenation using near-infrared spectroscopy in preterm infants with symptomatic anemia. Transfusion 54, 1100-1105 (2014).

68. Seidel, D. et al. Changes in regional tissue oxygenation saturation and desaturations after red blood cell transfusion in preterm infants. J. Perinatol. 33, 282-287 (2013).

69. van Hoften, J. C. R., Verhagen, E. A., Keating, P., ter Horst, H. J. \& Bos, A. F. Cerebral tissue oxygen saturation and extraction in preterm infants before and after blood transfusion. Arch. Dis. Child Fetal Neonatal Ed. 95, F352-F358 (2010).

70. Wardle, S. P., Garr, R., Yoxall, C. W. \& Weindling, A. M. A pilot randomised controlled trial of peripheral fractional oxygen extraction to guide blood transfusions in preterm infants. Arch. Dis. Child Fetal Neonatal Ed. 86, F22-F27 (2002).

71. Wardle, S. P., Yoxall, C. W., Crawley, E. \& Weindling, A. M. Peripheral oxygenation and anemia in preterm babies. Pediatr. Res. 44, 125-131 (1998).

72. White, L., Said, M. \& Rais-Bahrami, K. Monitoring mesenteric tissue oxygenation with near-infrared spectroscopy during packed red blood cell transfusion in preterm infants. J. Neonatal Perinatal Med 8, 157-163 (2015).

73. Whitehead, H. V., Vesoulis, Z. A., Maheshwari, A., Rao, R. \& Mathur, A. M. Anemia of prematurity and cerebral near-infrared spectroscopy: should transfusion thresholds in preterm infants be revised? J. Perinatol. 38, 1022-1029 (2018).

74. Saito-Benz, M., Sandle, M. E., Jackson, P. B. \& Berry, M. J. Blood transfusion for anaemia of prematurity: current practice in Australia and New Zealand. J. Paediatr. Child Health https://doi.org/10.1111/jpc.14222 (2018).

75. Bailey, S. M., Hendricks-Munoz, K. D. \& Mally, P. V. Variability in splanchnic tissue oxygenation during preterm red blood cell transfusion given for symptomatic anaemia may reveal a potential mechanism of transfusion-related acute gut injury. Blood Transfus. 13, 429-434 (2015).

76. Marin, T., Josephson, C. D., Kosmetatos, N., Higgins, M. \& Moore, J. E. Feeding preterm infants during red blood cell transfusion is associated with a decline in postprandial mesenteric oxygenation. J. Pediatr. 165, 464-471 e1 (2014).

77. Marin, T. et al. Red blood cell transfusion-related necrotizing enterocolitis in very-low-birthweight infants: a near-infrared spectroscopy investigation. Transfusion 53, 2650-2658 (2013).

78. Marin, T. \& Moore, J. E. Mesenteric oxygenation changes associated with necrotizing enterocolitis and pneumoperitoneum after multiple blood transfusions: a case report. Adv. Neonatal Care 18, 121-127 (2018).

79. Clyman, R. I. Patent ductus arteriosus, its treatments, and the risks of pulmonary morbidity. Semin. Perinatol. 42, 235-242 (2018).

80. Hundscheid, T. et al. Early treatment versus expectative management of patent ductus arteriosus in preterm infants: a multicentre, randomised, non-inferiority trial in Europe (BeNeDuctus trial). BMC Pediatr. 18, 262 (2018).

81. Smith, A., McNamara, P. J. \& El-Khuffash, A. F. Non-pharmacological management of a hemodynamically significant patent ductus arteriosus. Semin. Fetal Neonatal Med. 23, 245-249 (2018).

82. Benitz, W. E. Hey, doctor, leave the PDA alone. Pediatrics 140, e20170566 (2017).

83. Bixler, G. M., Powers, G. C., Clark, R. H., Walker, M. W. \& Tolia, V. N. Changes in the diagnosis and management of patent ductus arteriosus from 2006 to 2015 in United States neonatal intensive care units. J. Pediatr. 189, 105-112 (2017).

84. Hagadorn, J. I. et al. Covariation of neonatal intensive care unit-level patent ductus arteriosus management and in-neonatal intensive care unit outcomes following preterm birth. J. Pediatr. 203, 225-233 e1 (2018).

85. Kluckow, M. et al. A pilot randomised blinded placebo-controlled trial of paracetamol for later treatment of a patent ductus arteriosus. J. Perinatol. https://doi. org/10.1038/s41372-018-0247-z (2018).

86. Liebowitz, M. \& Clyman, R. I. Prophylactic indomethacin compared with delayed conservative management of the patent ductus arteriosus in extremely preterm infants: effects on neonatal outcomes. J. Pediatr. 187, 119-126 e1 (2017).

87. Louis, D. et al. Predictors of PDA treatment in preterm neonates who had received prophylactic indomethacin. Am. J. Perinatol. 35, 509-514 (2018).

88. Sallmon, H., Koehne, P. \& Hansmann, G. Recent advances in the treatment of preterm newborn infants with patent ductus arteriosus. Clin. Perinatol. 43, 113-129 (2016).

89. Yap, N. \& Bharucha, T. Patent ductus arteriosus in extreme prematurity: role of echocardiography and other imaging techniques. Curr. Pediatr. Rev. 12, 126-135 (2016). 
90. Thompson, E. J. et al. Association between furosemide exposure and patent ductus arteriosus in hospitalized infants of very low birth weight. J. Pediatr. 199, 231-236 (2018).

91. Chock, V. Y., Rose, L. A., Mante, J. V. \& Punn, R. Near-infrared spectroscopy for detection of a significant patent ductus arteriosus. Pediatr. Res. 80, 675-680 (2016).

92. Petrova, A., Bhatt, M. \& Mehta, R. Regional tissue oxygenation in preterm born infants in association with echocardiographically significant patent ductus arteriosus. J. Perinatol. 31, 460-464 (2011).

93. Schwarz, C. E., Preusche, A., Wolf, M., Poets, C. F. \& Franz, A. R. Prospective observational study on assessing the hemodynamic relevance of patent ductus arteriosus with frequency domain near-infrared spectroscopy. BMC Pediatr. 18, 66 (2018).

94. Underwood, M. A., Milstein, J. M. \& Sherman, M. P. Near-infrared spectroscopy as a screening tool for patent ductus arteriosus in extremely low birth weight infants. Neonatology 91, 134-139 (2007).

95. van der Laan, M. E. et al. A hemodynamically significant patent ductus arteriosus does not affect cerebral or renal tissue oxygenation in preterm infants. Neonatology 110, 141-147 (2016).

96. Chock, V. Y., Ramamoorthy, C. \& Van Meurs, K. P. Cerebral oxygenation during different treatment strategies for a patent ductus arteriosus. Neonatology 100, 233-240 (2011).

97. Dani, C. et al. Effect on cerebral oxygenation of paracetamol for patent ductus arteriosus in preterm infants. Eur. J. Pediatr. 177, 533-539 (2018).

98. Lemmers, P. M., Molenschot, M. C., Evens, J., Toet, M. C. \& van Bel, F. Is cerebral oxygen supply compromised in preterm infants undergoing surgical closure for patent ductus arteriosus? Arch. Dis. Child Fetal Neonatal Ed. 95, F429-F434 (2010).

99. Vanderhaegen, J. et al. Surgical closure of the patent ductus arteriosus and its effect on the cerebral tissue oxygenation. Acta Paediatr. 97, 1640-1644 (2008).

100. Goldstein, S. D. et al. Early detection of necrotizing enterocolitis using broadband optical spectroscopy. J. Pediatr. Surg. 53, 1192-1196 (2018).

101. Al-Hamad, S. et al. Contrast-enhanced ultrasound and near-infrared spectroscopy of the neonatal bowel: novel, bedside, noninvasive, and radiation-free imaging for early detection of necrotizing enterocolitis. Am. J. Perinatol. 35, 1358-1365 (2018)

102. DeWitt, A. G., Charpie, J. R., Donohue, J. E., Yu, S. \& Owens, G. E. Splanchnic nearinfrared spectroscopy and risk of necrotizing enterocolitis after neonatal heart surgery. Pediatr. Cardiol. 35, 1286-1294 (2014).

103. Patel, A. K. et al. Abdominal near-infrared spectroscopy measurements are lower in preterm infants at risk for necrotizing enterocolitis. Pediatr. Crit. Care Med. 15 735-741 (2014)

104. Schat, T. E. et al. Near-infrared spectroscopy to predict the course of necrotizing enterocolitis. PLOS ONE 11, e0154710 (2016).

105. Cortez, J. et al. Noninvasive evaluation of splanchnic tissue oxygenation using near-infrared spectroscopy in preterm neonates. J. Matern Fetal Neonatal Med. 24, 574-582 (2010).

106. Bozzetti, V. et al. Evaluation of splanchnic oximetry, Doppler flow velocimetry in the superior mesenteric artery and feeding tolerance in very low birth weight IUGR and non-IUGR infants receiving bolus versus continuous enteral nutrition. BMC Pediatr. 12, 106 (2012).

107. Gillam-Krakauer, M. et al. Correlation of abdominal $\mathrm{rSO}_{2}$ with superior mesenteric artery velocities in preterm infants. J. Perinatol. 33, 609-612 (2013).

108. Akotia, D. H., Durham, J. T., Arnell, K. M., Petruzzelli, D. L. \& Katheria, A. C. Relationship between near-infrared spectroscopy and transabdominal ultrasonography: noninvasive monitoring of intestinal function in neonates. Med Sci. Monit. 22, 61-68 (2016).

109. Bozzetti, V. et al. Impact of continuous vs bolus feeding on splanchnic perfusion in very low birth weight infants: a randomized trial. J. Pediatr. 176, 86-92 e2 (2016).

110. Grometto, A., Pizzo, B., Strozzi, M. C., Gazzolo, F. \& Gazzolo, D. Near-infrared spectroscopy is a promising noninvasive technique for monitoring the effects of feeding regimens on the cerebral and splanchnic regions. Acta Paediatr. 107, 234-239 (2018).

111. Corvaglia, L. et al. Bolus vs. continuous feeding: effects on splanchnic and cerebral tissue oxygenation in healthy preterm infants. Pediatr. Res. 76, 81-85 (2014).

112. Corvaglia, L. et al. Splanchnic oxygenation at first enteral feeding in preterm infants: correlation with feeding intolerance. J. Pediatr. Gastroenterol. Nutr. 64, 550-554 (2017)

113. Dani, $C$. et al. Splanchnic tissue oxygenation for predicting feeding tolerance in preterm infants. J. Parent. Enter. Nutr. 39, 935-940 (2015).

114. Bozzetti, V., Tagliabue, P. E., Visser, G. H., van Bel, F. \& Gazzolo, D. Feeding issues in IUGR preterm infants. Early Hum. Dev. 89(Suppl. 2), S21-S23 (2013).
115. Schat, T. E. et al. Abdominal near-infrared spectroscopy in preterm infants: a comparison of splanchnic oxygen saturation measurements at two abdominal locations. Early Hum. Dev. 90, 371-375 (2014).

116. Aly, S. A. et al. Cerebral tissue oxygenation index and lactate at $24 \mathrm{~h}$ postoperative predict survival and neurodevelopmental outcome after neonatal cardiac surgery. Congenit. Heart Dis. 12, 188-195 (2017).

117. Ferradal, S. L. et al. Non-invasive assessment of cerebral blood flow and oxygen metabolism in neonates during hypothermic cardiopulmonary bypass: feasibility and clinical implications. Sci. Rep. 7, 44117 (2017).

118. Kuik, S. J. et al. Preterm infants undergoing laparotomy for necrotizing enterocolitis or spontaneous intestinal perforation display evidence of impaired cerebrovascular autoregulation. Early Hum. Dev. 118, 25-31 (2018).

119. Mintzer, J. P. \& Messina, C. Cerebral oxygenation during umbilical arterial blood sampling in very low birth weight neonates. J. Perinatol. 38, 368-373 (2018).

120. Mintzer, J. P., Parvez, B. \& La Gamma, E. F. Umbilical arterial blood sampling alters cerebral tissue oxygenation in very low birth weight neonates. J. Pediatr. 167, 1013-1017 (2015).

121. Roll, C., Huning, B., Kaunicke, M., Krug, J. \& Horsch, S. Umbilical artery catheter blood sampling decreases cerebral blood volume and oxygenation in very low birthweight infants. Acta Paediatr. 89, 862-866 (2000).

122. Roll, C., Huning, B., Kaunicke, M., Krug, J. \& Horsch, S. Umbilical artery catheter blood sampling volume and velocity: impact on cerebral blood volume and oxygenation in very-low-birthweight infants. Acta Paediatr. 95, 68-73 (2006).

123. Schulz, G. et al. Slow blood sampling from an umbilical artery catheter prevents a decrease in cerebral oxygenation in the preterm newborn. Pediatrics 111, e73-e76 (2003).

124. Ancora, G. et al. Effect of posture on brain hemodynamics in preterm newborns not mechanically ventilated. Neonatology 97, 212-217 (2010).

125. Demirel, G., Oguz, S. S., Celik, I. H., Erdeve, O. \& Dilmen, U. Cerebral and mesenteric tissue oxygenation by positional changes in very low birth weight premature infants. Early Hum. Dev. 88, 409-411 (2012).

126. Fyfe, K. L. et al. Preterm infants exhibit greater variability in cerebrovascular control than term infants. Sleep 38, 1411-1421 (2015).

127. Wong, F. et al. Cerebrovascular control is altered in healthy term infants when they sleep prone. Sleep 36, 1911-1918 (2013).

128. Bozzetti, V. et al. Cerebral and somatic NIRS-determined oxygenation in IUGR preterm infants during transition. J. Matern Fetal Neonatal Med. 29, 443-446 (2016).

129. Montaldo, P., De Leonibus, C., Giordano, L., De Vivo, M. \& Giliberti, P. Cerebral, renal and mesenteric regional oxygen saturation of term infants during transition. J. Pediatr. Surg. 50, 1273-1277 (2015).

130. Terstappen, F. et al. Elevated renal tissue oxygenation in premature fetal growth restricted neonates: an observational study. PLOS ONE 13, e0204268 (2018).

131. Bailey, S. M., Hendricks-Munoz, K. D. \& Mally, P. Cerebral, renal, and splanchnic tissue oxygen saturation values in healthy term newborns. Am. J. Perinatol. 31, 339-344 (2014).

132. McNeill, S., Gatenby, J. C., McElroy, S. \& Engelhardt, B. Normal cerebral, renal and abdominal regional oxygen saturations using near-infrared spectroscopy in preterm infants. J. Perinatol. 31, 51-57 (2010).

133. Tataranno, M. L. et al. Early oxygen-utilization and brain activity in preterm infants. PLOS ONE 10, e0124623 (2015).

134. Cohen, E. et al. Cerebrovascular autoregulation in preterm fetal growth restricted neonates. Arch. Dis. Child Fetal Neonatal Ed. https://doi.org/10.1136/ archdischild-2017-313712 (2018).

135. Thewissen, L. et al. Measuring near-infrared spectroscopy derived cerebral autoregulation in neonates: from research tool toward bedside multimodal monitoring. Front. Pediatr. 6, 117 (2018).

136. Ono, M. et al. Risks for impaired cerebral autoregulation during cardiopulmonary bypass and postoperative stroke. Br. J. Anaesth. 109, 391-398 (2012).

137. Soul, J. S. et al. Fluctuating pressure-passivity is common in the cerebral circulation of sick premature infants. Pediatr. Res 61, 467-473 (2007).

138. Alderliesten, T. et al. Cerebral oxygenation, extraction, and autoregulation in very preterm infants who develop peri-intraventricular hemorrhage. J. Pediatr. 162, 698-704 e2 (2013).

139. Ono, M. et al. Duration and magnitude of blood pressure below cerebral autoregulation threshold during cardiopulmonary bypass is associated with major morbidity and operative mortality. J. Thorac. Cardiovasc. Surg. 147, 483-489 (2014).

140. Verhagen, E. A., Hummel, L. A., Bos, A. F. \& Kooi, E. M. Near-infrared spectroscopy to detect absence of cerebrovascular autoregulation in preterm infants. Clin. Neurophysiol. 125, 47-52 (2014).

141. Dix, L. M., van Bel, F. \& Lemmers, P. M. Monitoring cerebral oxygenation in neonates: an update. Front. Pediatr. 5, 46 (2017). 
142. Zhang, Y. et al. Cerebral near-infrared spectroscopy analysis in preterm infants with intraventricular hemorrhage. Conf. Proc. IEEE Eng. Med. Biol. Soc. 2011, 1937-1940 (2011).

143. Greisen, G., Andresen, B., Plomgaard, A. M. \& Hyttel-Sorensen, S. Cerebral oximetry in preterm infants: an agenda for research with a clear clinical goal. Neurophotonics 3, 031407 (2016).

144. Hyttel-Sorensen, S. et al. Clinical use of cerebral oximetry in extremely preterm infants is feasible. Dan. Med. J. 60, A4533 (2013).

145. Clair, M. P. et al. Prognostic value of cerebral tissue oxygen saturation during neonatal extracorporeal membrane oxygenation. PLOS ONE 12, e0172991 (2017).

146. Lau, P. E. et al. Use of renal near-infrared spectroscopy measurements in congenital diaphragmatic hernia patients on ECMO. J. Pediatr. Surg. 52, 689-692 (2017)

147. Tian, F., Jenks, C., Potter, D., Miles, D. \& Raman, L. Regional cerebral abnormalities measured by frequency-domain near-infrared spectroscopy in pediatric patients during extracorporeal membrane oxygenation. ASAIO J. 63, e52-e59 (2017).

148. Adams, P. S. et al. Associations of perioperative renal oximetry via near-infrared spectroscopy, urinary biomarkers, and postoperative acute kidney injury in infants after congenital heart surgery: should creatinine continue to be the gold Standard? Pediatr. Crit. Care Med. https://doi.org/10.1097/PCC.0000000000001767 (2018).

149. Balci, C., Haftaci, E. \& Kunt, A. T. Use of cerebral oxygen saturation and hemoglobin concentration to predict acute kidney injury after cardiac surgery. J. Int. Med. Res. 46, 1130-1137 (2018).

150. Ersoy, C. et al. Are perioperative near-infrared spectroscopy values correlated with clinical and biochemical parameters in cyanotic and acyanotic infants following corrective cardiac surgery? Perfusion 31, 125-130 (2016).

151. Hickok, R. L., Spaeder, M. C., Berger, J. T., Schuette, J. J. \& Klugman, D. Postoperative abdominal NIRS values predict low cardiac output syndrome in neonates. World J. Pediatr. Congenit. Heart Surg. 7, 180-184 (2016).

152. Chakravarti, S. B. et al. Multisite near-infrared spectroscopy predicts elevated blood lactate level in children after cardiac surgery. J. Cardiothorac. Vasc. Anesth. 23, 663-667 (2009).

153. Hoffman, G. M. et al. Postoperative cerebral and somatic near-infrared spectroscopy saturations and outcome in hypoplastic left heart syndrome. Ann. Thorac. Surg. 103, 1527-1535 (2017).

154. Sood, E. D., Benzaquen, J. S., Davies, R. R., Woodford, E. \& Pizarro, C. Predictive value of perioperative near-infrared spectroscopy for neurodevelopmental outcomes after cardiac surgery in infancy. J. Thorac. Cardiovasc. Surg. 145, 438-445e1 (2013). discussion 44-5.

155. Spaeder, M. C. et al. Perioperative near-infrared spectroscopy monitoring in neonates with congenital heart disease: relationship of cerebral tissue oxygenation index variability with neurodevelopmental outcome. Pediatr. Crit. Care Med. 18, 213-218 (2017).

156. Colasacco, C., Worthen, M., Peterson, B., Lamberti, J. \& Spear, R. Near-infrared spectroscopy monitoring to predict postoperative renal insufficiency following repair of congenital heart disease. World J. Pediatr. Congenit. Heart Surg. 2, 536-540 (2011).

157. Ruf, B. et al. Intraoperative renal near-infrared spectroscopy indicates developing acute kidney injury in infants undergoing cardiac surgery with cardiopulmonary bypass: a case-control study. Crit. Care 19, 27 (2015).

158. Lin, N., Flibotte, J. \& Licht, D. J. Neuromonitoring in the neonatal ECMO patient. Semin. Perinatol. 42, 111-121 (2018).

159. Koch, H. W. \& Hansen, T. G. Perioperative use of cerebral and renal near-infrared spectroscopy in neonates: a 24-h observational study. Paediatr. Anaesth. 26, 190-198 (2016).

160. Beck, J. et al. Monitoring cerebral and renal oxygenation status during neonatal digestive surgeries using near infrared spectroscopy. Front. Pediatr. 5, 140 (2017).

161. Conforti, A. et al. Near infrared spectroscopy: experience on esophageal atresia infants. J. Pediatr. Surg. 49, 1064-1068 (2014).

162. Stolwijk, L. J. et al. Brain oxygenation during thoracoscopic repair of long gap esophageal atresia. World J. Surg. 41, 1384-1392 (2017).

163. Tina, L. G. et al. Near infrared spectroscopy in healthy preterm and term newborns: correlation with gestational age and standard monitoring parameters. Curr. Neurovasc. Res. 6, 148-154 (2009).

164. Hunter, C. L., Oei, J. L., Lui, K. \& Schindler, T. Cerebral oxygenation as measured by near-infrared spectroscopy in neonatal intensive care: correlation with arterial oxygenation. Acta Paediatr. 106, 1073-1078 (2017).

165. Wintermark, P., Hansen, A., Warfield, S. K., Dukhovny, D. \& Soul, J. S. Nearinfrared spectroscopy versus magnetic resonance imaging to study brain perfusion in newborns with hypoxic-ischemic encephalopathy treated with hypothermia. Neuroimage 85(Part 1), 287-293 (2014).

166. Bernal, N. P., Hoffman, G. M., Ghanayem, N. S. \& Arca, M. J. Cerebral and somatic near-infrared spectroscopy in normal newborns. J. Pediatr. Surg. 45, 1306-1310 (2010).

167. Baik, N. et al. Reference ranges for cerebral tissue oxygen saturation index in term neonates during immediate neonatal transition after birth. Neonatology 108, 283-286 (2015). 